\title{
DIGITIZATION SOFTWARE OBSOLESCENCE, TOO?
}

Dave Rice, City University of New York (CUNY), USA

\section{Introduction}

Audiovisual archivists have long been aware of the impending obsolescence issues surrounding videotape and its associated hardware. The machines, skills, and technological environments that support tape-based video formats evaporate as the larger communities of video broadcasting and production move on to file-based digital environments. Archives are increasingly challenged to sustain local means to access and preserve videotape collections. Although the obsolescence of hardware has been a primary concern, videotape digitization is also dependent on software to facilitate a migration or transformation of data from a videotape to a digital file. The obsolescence of videotape digitization software itself is a secondary technological challenge.

Digitization software, like most video hardware, is designed for and marketed to the larger communities that produce or broadcast audiovisual content. Audiovisual archivists have often adopted such software despite the limitations these present within an archival context. The objectives, methods, and challenges faced by an audiovisual archivist working with deteriorated videotape content are significantly different than the objectives of the production assistant digitizing segments from tape to use in an edit. With a few exceptions, digitization software is streamlined for production use but cumbersome for preservation use. As developers of video digitization software follow their larger user communities away from videotape workflows, archivists encounter obsolescence risks approaching from both sides; as the machines, skills, and services for video hardware become less available, digitization software also deprecates. This downtrend in the market for digitization software for videotape foretells the obsolescence of digitization software at a rate comparable to videotape itself. Just as archivists are challenged to cope with hardware where limited and lessening support is available, this is increasingly true of software.

Although videotape digitization software options may dwindle, other technological advances make other aspects of videotape digitization much more efficient and flexible. Until recently, capturing high quality video required specialized hardware and computers. The high data rates of uncompressed video and audio limited what types of connectivity could be used with videotape digitization. Today's faster connections such as Thunderbolt and USB3 have no trouble transporting uncompressed video at the playback speed, and more modern computers are capable of supporting audiovisual digitization. Despite impending software obsolescence issues, the digitization of archival audiovisual material is increasingly more approachable now than before as digitization systems may now be built without requiring the highest end of dedicated computers.

\section{Digitization Software Options for AV ${ }^{6}$}

Software that facilitates migration of audiovisual data from tape to file is developed under a variety of circumstances: some by hardware developers to demonstrate the value and function of their products; some for a more comprehensive set of functions targeted at content creators and editors; some to facilitate the migration of data from a particular type of digital tape to a file; and some within the context of an open source project. Naturally there is overlap between these categories, but in general these categories affect software obsolescence in distinct ways.

6 The software referenced in this section is not intended to be either a comprehensive list or an endorsement, but provided in order to detail appropriate examples of the objectives and development circumstances where audiovisual digitization software can occur. 


\section{Specialty Software for Specific Tape Formats}

This category includes both proprietary and open source software designed to migrate data from specific digital tape formats. Examples of this software include Live Capture Plus and dvgrab, used for migrating data from DV tape via FireWire, as well as DATXtract, readdat, and ReadDAT X, used for migrating encapsulated PCM audio data from DAT tape via SCSI. This type of software often provides significant efficiencies for the preservation of certain forms of digital tape by focusing on verifiable migration of data rather than digitization. Both DAT and DV tape utilize parity data that may be used by software to assess the accuracy of the data migration. ${ }^{7}$ The specifications of the digital formats written on digital tape play a central role in the design and features of this type of software.

Since most data tape formats are no longer used (with the exception of LTO), specialty reading software for these formats have mostly been abandoned. Currently none of the graphical software to read DAT tapes in Mac OS X supports modern Intel processors and instead requires older PowerPC Macs. Documentation for all DAT-reading software is increasingly available solely via the Internet Archive as link rot affects the online information of these tools. ${ }^{8}$ While there is still an active Google Group focused on data migration software for DAT tape, datheads 2 wav, overall such resources for this approach are greatly depleted.

As the use of MiniDV has virtually ceased for recording, the availability of software to transfer MiniDV has been compromised. Some of the most effective software at migrating MiniDV tape, such as Live Capture Plus and dvgrab, are still available online but have not been updated in over 5 years. Squarebox's website lists Live Capture Plus as a “Legacy product - Not recommend for new installations [sic]." In May 2013 the website of the KinoVideo Editor, the parent project of dvgrab, listed Kino as "a dead project"10 and had closed discussion forums.

Betacam SX records a 4:2:2 MPEG-2 stream onto digital tape. For a short time SONY sold the DNE-700 Digital Editing System", which included software to facilitate the transfer of MPEG-2 data from Betacam SX to an attached server. The software has been largely unavailable for about 15 years, and no other significant software workflows have been developed to transfer MPEG-2 from Betacam SX to file. Currently the most common migration workflow for Betacam SX in a digital preservation environment is the use of a Betacam SX deck to transcode the MPEG-2 on the tape to SDI for subsequent conversion to a file ${ }^{12}$.

\section{Editing / Production Software}

Software designed for editing and production is generally the most visible option for videotape digitization with support from the largest communities and resources. Products such as Adobe Premiere and Final Cut Pro were developed by large companies for broad user communities. Video editing software is generally complex and full-featured, while videotape digitization is just a minor component.

Final Cut Pro 7's Log and Capture feature served as a ubiquitous tool in the Mac OS environment for videotape digitization, but the software has not been officially supported by Apple for over 4 years. Some archivists have worked to sustain Final Cut Pro 7 as a means of digitization; however, Apple's transition from 32-bit QTKit to the 64-bit AVFoundation has been a substan-

7 DatXtract will log parity verification errors during data migration. Live Capture Plus record a DV stream from tape to file over Firewire so that the resulting stream may be analyzed for error concealment codes.

8 As an example, see the Internet Archive's capture of Ade Rixon's collection of DAT/DDS info at http://web. archive.org/web/20070828045540/http://homepage.ntlworld.com/adrian.rixon/personal/ade/dat-dds/index.html

$9 \mathrm{http}: / / \mathrm{www} \cdot$ squarebox.com/legacy/intlstore.html

10 http://www.kinodv.org/article/archive/0

I I https://web.archive.org/web/2006050922484l/http://www.pmotions.com/Public/PDFs/SONY/DNE-700.pdf

12 An hour of MPEG-2 on Betacam SX tape is roughly 8 gigabytes of compressed data. Once decoded and converted to $\mathrm{SDI}$ the same hour is roughly 100 gigabytes. 
tial revision of Mac OS X's media handling features, which does not prioritize sustaining legacy hardware and workflows. Final Cut Pro X, which supersedes Final Cut Pro 7, does not include the majority of the videotape functions that its predecessor did. Additionally, as Apple's QTKit is phased out in Mac operating system updates, the Log and Transfer interface of Final Cut Pro 7 has become increasingly unreliable on the later versions of the operating system. Some archives that rely on Final Cut Pro 7 for digitization have experienced significant issues as a side effect of upgrading their operating system, often compelling them to revert back to older, unsupported operating systems. Just as the maintenance of most videotape hardware requires the utilization of unofficial, unsupported, and/or creative means to acquire legacy machinery and skills, the maintenance of digitization software is increasingly in need of similar alternative strategies.

\section{Software Accompanying Hardware}

The hardware that converts analog or digital video signals to computer inputs, such as the BlackMagic Ultrastudio Express, the AJA io, and OpenCube ingest servers, are accompanied by software that demonstrates their features and facilitates system diagnosis. Digitization hardware from BlackMagic is typically accompanied by software like Media Express, while AJA provides VTR Xchange. Though these applications are not open source and supported only on their associated hardware, both are freely available.

The benefit of this type of software is the ease of use. Both Media Express andVTR Xchange are designed to offer a demonstrative and intuitive user experience, and such applications are entirely focused on transferring audiovisual data between tapes and computers. However, the simplicity of these applications means the options for fine-tuning are limited. For instance, Media Express provides no options to control audio bit depth, allowing only 16 bit audio during recording, even though the hardware itself supports 32 bit audio. Compared to editing software, there are fewer options for audio channel configuration, display aspect ratio handling, and the utilization of codecs. Although this category of software places arbitrary limits on recording options, such software may allow for more efficient workflows.

\section{Open Source Projects}

The Österreichische Mediathek has released DVA Profession ${ }^{13}$, an open source digitization solution combining tools and workflows for videotape digitization, access, review, and management of digitization projects. DVA Profession facilitates the digitization of videotape by combining several open source projects together in a Windows environment. The approach uses ffdshow-tryouts ${ }^{14}$ which adds open source codec libraries as system codecs. With ffdshowtryouts enabling lossless video codecs at a system level, DVA Profession then uses VirtualDub ${ }^{15}$ to facilitate the ingest from SDI or DV inputs through an encoding process to create a representative preservation file. DVA Profession provides scripts to automatically setup VirtualDub according to its preservation recommendations, such as setting the recordings specifications appropriate for the selected video input.

BlackMagic Design, which designs and sells hardware to facilitate the use of audiovisual signals as computer inputs, makes their Software Development Kit (SDK) freely available under an non-restrictive license. ${ }^{16}$ Use of the SDK requires an end user license agreement but does not require a non-disclosure agreement or complex partnership as is often required with other hardware vendors. Blackmagic's SDK has enabled many active, open source projects to inte-

$13 \mathrm{http}: / / w w w . d v a-p r o f e s s i o n . m e d i a t h e k . a t /$

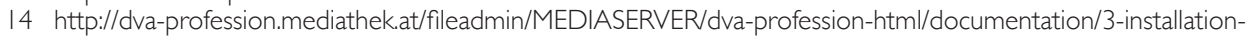
manual/32-ingest-clients/index.html\#c3 I I http://ffdshow-tryout.sourceforge.net/

15 http://dva-profession.mediathek.at/fileadmin/MEDIASERVER/dva-profession-html/documentation/3-installationmanual/32-ingest-clients/index.html\#c312 http://virtualdub.org/

16 + I Blackmagic Design 
grate with Blackmagic hardware, such as VLC, Shotcut, and FFmpeg. Beyond Blackmagic, many other devices have been integrated into multimedia software frameworks such as FFmpeg. ${ }^{17}$ FFmpeg is a comprehensive set of open sources utilities and libraries for handling audiovisual data, including analysis, encoding, decoding, metadata handling, and audiovisual processing. See http://ffmpeg.org/ffmpeg-devices.htm|\#Input-Devices for examples and information on FFmpeg's integration with hardware devices.

While the Blackmagic SDK is a significant building block towards the development of open source videotape digitization software, the libraries and proof-of-concept utilities that comprise the SDK are intended for programmers and developers rather than archivists and preservationists. To facilitate the integration between video hardware, via Blackmagic capture products, and audiovisual frameworks, such as Libav and FFmpeg, there is bmdtools. ${ }^{18}$ Bmdtools includes bmdplay and bmdcapture which make the use of the Blackmagic SDK considerably easier. With bmdcapture an operator can select both audio and video bit depth as well as audio channel count, signal standard, and input connection, and send all the resulting data into another tool such as Libav or FFmpeg for further transcoding or playback while recording to a file. Bmdtools enables the use of Blackmagic hardware with substantially more options and flexibly as compared with BlackMagic's own MediaExpress software.

During a Hack Day hosted at the 2014 conference of the Association of Moving Image Archivists, one team focused on the creation of fully functional digitization software. ${ }^{19}$ The team combined skills to integrate bmdtools ${ }^{20}$, QCTools ${ }^{21}$, Blackmagic SDK ${ }^{22}$, and ffmpeg ${ }^{23}$ within the framework of an XCode project. By the vote of the conference attendees, the project won top honors at the competitive Hack Day by demonstrating the need and interest in the design of videotape digitization software based on open source components. ${ }^{24}$

Also in 2014, the Bay Area Video Coalition (BAVC) applied for and won a competitive Knight Foundation Prototype Grant to research, design, and build an open source application to digitize videotape. ${ }^{25}$ The work resulted in the integration of the Blackmagic SDK into their open source quality control application, QCTools. An experimental feature of QCTools can detect Blackmagic hardware and subsequently offer additional options for video capture. After configuring the specifications of the capture, the recording will start and the digitization will render original time-based analytical graphs and thumbnails for review. ${ }^{26}$ Presently this feature is still in experimental status and requires optimization and refinements for memory management. The project also resulted in contributions to vrecord ${ }^{27}$, a bash script that coordinates the Blackmagic SDK, bmdtools, QCTools and FFmpeg to record videotapes to uncompressed and lossless formats with logging, metadata, and fixity data.

I7 FFmpeg is an open source solution to record, transcode, process and analyze audio and video. Its codec and format libraries are widely incorporated into audiovisual software.

18 bmdtools, developed by Luca Barbato is available, at https://github.com/lu-zero/bmdtools

19 Disclosure: the principal author of this document was a member of this Hack Day team

20 http://github.com/lu-zero/bmdtools

$21 \mathrm{http}: / /$ www.bavc.org/qctools

22 http://software.blackmagicdesign.com/SDK/Blackmagic_DeckLink_SDK_I 0.I.4.zip

23 http://ffmpeg.org

24 Additional info about the project outcome is avialable at http://wiki.curatecamp.org/index.php/Association_of_ Moving_Image_Archivists_\%26_Digital_Library_Federation_Hack_Day_20 I4\#Hack_day_capture:_GUI_tool_ for_BMDCapture.2C_using_FFmpeg_.2B_BMDTools_2B_BlackMagic_Decklink_SDK and https://github.com/ amiaopensource/hackdaycapture

25 http://www.bavc.org/bavc-preservation-awarded-knight-foundation-prototype-fund http://www.knightfoundation. org/grants/201449/23/

26 Details and images of this feature in QCTools may be reviewed here: http://htmlpreview.github.io/?https:/github. $\mathrm{com} / \mathrm{bavc/qctools/blob/master/Source/Resource/Help/Recording/Recording.html}$

27 vrecord is available at http://github.com/amiaopensource/vrecord/blob/master/vrecord 


\section{Open Sourcing Digitization}

The obsolescence of audiovisual digitization software presents both a challenge and an opportunity. Events such as the end-of-support for software, Apple's transition from QtKit to AVFoundation (and the associated rewrites to QuickTime Pro $X$ and Final Cut X), and the closure of discussion forums related to digitization software, signal that archivists should collectively take further control of the software required for digitization. In recent years there has been increased communal efforts, funding, and progress at building tools for cataloging, processing, collection management, and providing online access to video. The embrace of "open source" within the preservation community has been a blessing, as it has become increasingly easy to share resources, customize existing solutions for local challenges, and solve problems communally. Resources such as JHOVE, Archivematica, Omeka, Medialnfo, BWF MetaEdit and bagit have been developed under open licenses with the participation of archivists and have since found wide-scale deployment.

The values of open source software development are particularly relevant in the creation of software for preservation purposes. Whereas some software may be free in cost, open source software allows freedom for modification, invention, and collaboration. In a similar way to how archivists share workflows for videotape baking or videodeck modifications to meet preservation challenges, the same level of flexibility and creativity is needed software.

Relative to other archival open source projects, software that focuses on transferring content from tape to file has been sorely in need of open and participatory development specifically for preservation challenges. The development of such software is partly challenged by its reliance on hardware; video connections such as SDI, Component, Composite, or S-Video are not natively supported by common operating systems but require hardware and software working in conjunction to process incoming audiovisual signals to a resulting set of files. An open source digitization application may not be able to support all computer video hardware, but could start with support for what hardware is already supported by an open SDK or API.

\section{Designing an Audiovisual Digitization Application}

\section{Digitization Decision-Making}

In order to develop a potentially comprehensive open source approach for archival videotape digitization, there are several dialogues within the community to consider and respond to. Archival communities that focus on formats such as documents, still images, and audio have had longer experiences with digitization workflows, whereas the digitization of video (hampered by storage sizes, bandwidth, and expenses) has only recently become more approachable. While digitization practices for documents, still images, and audio include more community consensus regarding best practices and specifications, there is a much greater technical diversity regarding the workflows, specifications, and even objectives for digitizing archival video.

Audiovisual archives face a dilemma in which amassed collections of videotape require detailed processing and digitization through legacy hardware and (increasingly) legacy software in order to sustain collections. As archives commit to digitization and work to find support, funding, and momentum for these efforts a number of technical decisions and details must be established, including simple questions such as: What codec to use? What file format to use? What metadata? What specifications? And more complex questions: Should the technical discrepancies within a tape collection be replicated within the resulting files? Should audio channels be grouped into audio tracks fitting with the possible multiple audio presentations supported by the videotape? What metadata concerning the videotape, its content, or the digitization event should be stored in the resulting file?

The audiovisual archiving community has a rich and well-established professional body of skills and knowledge related to all aspects of analog video preservation, but a relatively new relationship with digital video. Within discussion, planning, and project management it is natural to 
focus on the details that are well understood and can be clearly debated and justified; however, often the most significant or costly decisions in project planning are the ones that are the least technically understood.

Parkinson's Law of Triviality, commonly referred to as bikeshedding, refers to scenarios where relatively unimportant aspects of planning receive abundant levels of focus and debate while more crucial details are skimmed over. Bikeshedding may be used to focus on what knowledge is known instead of what knowledge is needed. As archivists have often relied on the utilization of software for digitization and preservation work that was originally designed for production use, decision-making and planning for digitization and preservation efforts is occasionally limited to the context of this production software (such as envisioning specifications for a digitization plan based on what options are shown in Final Cut Pro 7). As archivists face greater technological challenges in preserving videotape content, there is less time to employ either a 'wait and see' or bikeshedding approach. Research, development, and informed action are sorely needed to reduce the risk of challenges impacting videotape digitization. The development of open source software, guided by the collaboration and participation of archivists, can play a central role in overcoming such challenges.

\section{Preservation Singularity and Diversity}

Although audiovisual archives have a rich history of managing technically diverse collections of multiple video formats used under various standards and specifications, there is often a recommendation to determine a singular digital specification for all digitization exercises. The rationale here is that the skill, knowledge, and hardware demanded to manage analog collections could be vastly simplified and reduced by requiring the archive to only manage one technical specification.

In practice this approach can appear to work in highly homogeneous collections, but within more technically diverse collections it can lead to compromises. For instance, to digitize all videotape to only a stereo audio configuration could create an unintended presentation where the tape may have a separate recordings or various language on different channels that are not intended for a left / right stereo presentation. To force all digitization to use four channel audio would be wasteful for video tapes that only provide 2 channels and lossy to video tapes that use more than four. Digitizing collections of mixed standards, such as PAL and NTSC, to a singular standard creates loss as frames and pixels are dropped or duplicated, and resized. Ideally digitization planning - and digitization software - should consider the significant characteristics of the diverse audiovisual collection being digitized in order to adapt digitization methods to best accommodate and maintain the significant properties throughout the process.

In the same way an archive may strive to ensure that it maintains all video decks necessary for access to the videotape collection, the same must be true for the digital counterparts. U-matic and Betacam SP were once considered suitable long-term carriers for video but continuing to sustain video in these carriers today is increasingly a sustainability risk. Likewise, decisionmaking for digital carriers made today may be tomorrow's risk and require reformatting similar to current practices for migrating analog material.

Archives should work to ensure sustained control of their adopted digital formats in order to maintain the necessary software to properly decode, present, and transcode the material efficiently, file-by-file or in large-scale batches of files. In the analog equivalent, this control was sustained through maintaining video decks. The players for digital video files are predominantly software based and have the advantage of often being open source and easily recreated and reproduced. ${ }^{28}$

28 The author has written further on the advantages and challenges of digital video players in a research paper for the Tate Museum available at http://www.tate.org.uk/research/publications/sustaining-consistent-video-presentation. 


\section{Standardization and Significant Characteristics}

To the greatest extent feasible the resulting digital file should mimic the significant characteristics of the analog source. Some significant characteristics are intrinsic to the analog format and some are interpreted and applied by an operator as the video is presented. Because of this, the selection of a target format is based partly on sustaining some characteristics (such as frame rate or field dominance) and clarifying other characteristics (such as aspect ratio and audio channel mapping).

Any effort to digitize videotape materials for the purposes of preservation should critically consider how the significant characteristics are sustained or compromised throughout the digitization process. In some instances an archivist may intentionally change one or more of these characteristics but should document these changes and take care not to do so unknowingly. Maintaining such characteristics ensures that the digital results are more authentic to the original and more effectively ensures that the resulting digital file is capable of reproducing the quality, content, and function of the original videotape.

Some characteristics of modern audiovisual file formats are ambiguous in the context of analog video tapes. Concepts such as display aspect ratio or audio channel configurations may have suggested defaults (such as presuming $4 / 3$ for display aspect ratio or the first channel as left and second channel as right for audio), but often deviations from the norm were generated and possibly documented (perhaps on the tape's label). While the playback hardware could not read the tape's labeling, the operator could use this information to make adjustments.

The aspect ratio refers to the ratio of width to height of the presented frame size, commonly 4:3 or 16:9 though many others are feasible. Although a digitized frame may be sampled and encoded as 720 pixels wide and 576 pixels high, the aspect ratio could be 4:3, 16:9, or possibly another ratio. An analog tape may labeled as 16:9 or anamorphic and intended to be adjusted when played back from a video deck. In these cases the display aspect ratio can be adjusted so that instead of digitizing a tape to $720 \times 576$ at $4: 3$ display aspect ratio it can be digitized to $720 \times 576$ at 16:9 display aspect ratio. Often the individual video pixels of a video frame are not intended to represent squares but rectangles (the ratio of the width and height of the pixels' shape whether rectangular or square is known as the pixel aspect ratio). If a $720 \times 576$ video frame is intended to be presented at a 4:3 display aspect ratio than those video pixels are tall and thin rectangles. If a $720 \times 576$ frame is intended to be presented at a 16:9 display aspect ratio than the pixels are short and wide rectangles. If a $720 \times 576$ video frame is presented with square pixels than the display aspect ratio would be 5:4 which is uncommon. The videotape contains the information that comprises the value and amount of pixels but it is a controllable, technical metadata value in the resulting file that determines what aspect ratio is used to present those pixels.

A video tape may have labeling to say the tape is stereo, or the first channel is mono audio only, or first channel for English and second channel for French. While the label relays information to an operator, it does not relay that data to a video deck. Thus, to present the videotape correctly requires some manual audio channel configuration.

Video digitization software often provides a means to arrange and label audio into tracks and channels to ensure that the intended audio presentation is supported by file metadata.

\section{Scenario \# I}

Here an analog video tape has 4 audio channels. The first two channels contain left and right presentations of a program and the third and fourth channels contain silence. The tables below outline two common tactics for digitizing this content. Option \#I maps each channel of the source tape to a channel of its own track and tags each track for mono presentation. During playback all four tracks present play simultaneously. This option does not clarify the audio channel arrangement within the resulting file. 


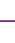

Option \#I

\begin{tabular}{|l|l|l|l|}
\hline Analog Channel & Content & Digital Track & Digital Channel \\
\hline Channel I & Program Left & Track I & Mono \\
\hline Channel 2 & Program Right & Track 2 & Mono \\
\hline Channel 3 & Silence & Track 3 & Mono \\
\hline Channel 4 & Silence & Track 4 & Mono \\
\hline
\end{tabular}

In option \#2 the first two channels are both mixed into a single two-channel stereo track so that the file on playback presents the tape's first channel for left presentation and second channel for right presentation and the other two channels of the video tape are not included in the digitization.

Option \#2

\begin{tabular}{|l|l|l|l|}
\hline Analog Channel & Content & Digital Track & Digital Channel \\
\hline Channel I & Program Left & Track I & Left \\
\hline Channel 2 & Program Right & Track I & Right \\
\hline Channel 3 & Silence & Unused & \\
\hline Channel 4 & Silence & Unused & \\
\hline
\end{tabular}

\section{Scenario \#2: Independent Content per Channel}

In this scenario a video tape includes distinct content on each channel. The channels are not intended to be presented simultaneously but are intended to be selected according to the intended presentation. If all channels were recorded to the same track (which is the only option given in Media Express) the presentation would be a cacophony of audio that is not intended for simultaneous presentation. In this scenario the audio channel mapping could be more clearly expressed as in the table below.

\begin{tabular}{|l|l|l|l|}
\hline Analog Channel & Content & Digital Track & Digital Channel \\
\hline Channel I & English & Track I & Mono (eng) \\
\hline Channel 2 & French & Track 2 & Mono (fre) \\
\hline Channel 3 & Spanish & Track 3 & Mono (spa) \\
\hline Channel 4 & Unused & Unused & \\
\hline
\end{tabular}

Although many digital audiovisual formats support language tags and descriptions for audio tracks $^{29}$ (which would greatly aid and clarify audiovisual presentation); unfortunately, these

29 It is worth noting that software for DVD authoring often provides mechanisms to add language tags and descriptions to various audio tracks, but it is worthwhile to incorporate and clarify this information within the digitization and preservation process as well. 
features are rarely integrated into existing videotape digitization software.$^{30}$ Additionally most videotape digitization software provides insufficient control over audio handling so that operators must map all of the videotape's audio channels to a single digital audio track. Final Cut Pro 7 provides some means to map audio channels into channel and track arrangements but also includes some burdensome limitations such as arbitrarily only handling a selection of 2 audio channels as an input from DV tape transmitted over FireWire when 4 audio channels may be present.

\section{Digitization Software Requirements}

Although the dream of a community-written, participatory, open source, audiovisual digitization software project is not yet fully realized, the building blocks for such an effort have been gradually falling into place. Many of those struggling with software in efforts to preserve videotapes know quite specifically the flaws of the process and what would relieve a significant amount of the burden and challenge of the process. An open source software effort could be as comprehensive as our preservation requirements are, including features such as:

- selection of audiovisual codecs to employ (with a focus on lossless and uncompressed)

- selection of audiovisual containers to employ

- audio channel mapping and configuration (to properly express audio arrangement)

- embedding of metadata or attachments that document the digitization process

- the ability to control key variable characteristics such as display aspect ratio

- strict adherence to the associated standards (such as expressing NTSC framerate as $30000 / 100$ I rather than 29.97 )

- incorporation with fixity, frame-checksum, and quality control features

- inclusion of scopes and analytics to supply real-time feedback on digitization quality

Open source, community-supported projects have been making inroads into other aspects of archival services. As the need for archives to support digitization efforts becomes more clear and essential, as other digitization software options become compromised, as the digitization preferences of the community become further standardized, and as the hardware manufacturers become more supportive of open source development, an open source approach to audiovisual digitization software is increasingly in demand for development, participation, and use.

Thanks to Athena Christa Holbrook, Kelly Haydon, Morgan Morel, Erik Piil, and Bertram Lyons. This work is licensed under a Creative Commons Attribution 4.0 International License ${ }^{31}$. 